\title{
Behaviour of a cloud of bubbles filled with vapour and a small amount of noncondensable gas (A theoretical and numerical study)
}

\author{
E. Klaseboer, G.J. de Bruin
}

Univ. of Twente, Enschede, Netherlands

\section{Nomenclature}

A radius of the bubble cloud $(m)$

$$
\text { velocity of sound }\left(\mathrm{m} \mathrm{sec}^{-1}\right)
$$

* specific velocity $\left(\mathrm{m} \mathrm{sec}^{-1}\right)$

$C_{p}$ spec. heat $\left(\mathrm{J} \mathrm{kg}^{-1} \mathrm{~K}^{-1}\right)$

$f$ pressure disturbance function

$\underline{\underline{I}}$ unit tensor

bubble number density

pressure $(\mathrm{Pa})$

$p_{a}$ applied pressure at the bubble cloud boundary $(\mathrm{Pa})$

$p_{r a}$ pressure due to the spherically symmetric outgoing waves $(\mathrm{Pa})$

$R$ radius of a bubble $(m)$

$r \quad$ radius in the bubbly mixture $(m)$

$\mathcal{R}$ universal ideal gas constant $\left(8.31 \mathrm{~Pa} \mathrm{~m}^{3} \mathrm{~mol}^{-1} \mathrm{~K}^{-1}\right)$ time (sec)

temperature $(K)$

velocity $\left(\mathrm{m} \mathrm{sec}^{-1}\right)$

dimensionless bubble mass $\alpha \quad$ void fraction

$\beta$ accommodation factor for condensation or evaporation $(\beta \approx 0.9)$

$\gamma \quad$ isentropic exponent of the vapour $(=1.4)$

$\varepsilon \quad$ ratio of velocities of sound in the mixture and in pure water

$\kappa$ constant factor

$\lambda$ thermal conductivity $\left(\mathrm{W} \mathrm{m}^{-1} \mathrm{~K}^{-1}\right)$

$\rho$ density $\left(\mathrm{kg} \mathrm{m}^{-3}\right)$

$\tau$ dimensionless time

$\chi \quad$ temperature potential

$\hat{x} \quad$ scaled value of quantity $x$.

\section{Subscripts :}

$b$ bubble

$e q$ equilibrium

$g$ gas

$l$ liquid

$m$ mixture gas/vapour

$v$ vapour

$o$ initial value.

Comportement d'un nuage de bulles remplies de vapeur et d'une petite quantité de gaz incondensable

La cavitation d'un nuage sphérique de bulles, remplies de vapeur et de gaz incondensable, a été étudiée. Des équations moyennées de mouvements à l'intérieur du nuage sont développées. En appliquant une perturbation de pression extérieure, la pression locale et le rayon d'une bulle à l'intérieur du nuage sont calculées. Deux fréquences, correspondant au comportement global d'un nuage et d'une bulle isolée, en résultent. Le comportement thermique passe de l'isothermique à l'adiabatique, en fonction de la quantité de gaz. 


\section{Introduction}

In cavitation a cloud of bubbles can separate from a propeller (fig. 1). The contents of the bubbles are unknown, it could be pure vapour or vapour with an amount of gas in it. A model for a completely gas filled bubbly cloud already exists (OMTA [1]). In this paper the behaviour of vapour bubble clouds and the difference with gas filled bubble clouds is investigated. A sudden pressure rise in the liquid surrounding a single bubble causes the bubble to collapse. A completely vapour filled bubble will show no rebound. If the bubble contains a few percent noncondensable gas, the bubble will rebound: gas is compressed in the final stage of the collapse. So even a small amount of noncondensable gas is of great importance! Some non-essential simplifications are made : spherical symmetric bubbles; condensation only occurs at the surface of a bubble; pressure is uniform within each bubble (PROSPERETTI [2]); damping is assumed to be caused mainly by thermal conduction and radiation of sound. If the radius of the bubbles is not too small and all velocities are small compared to the velocity of sound in the vapour, surface tension, viscosity and compressibility can be neglected. The bubbly cloud is also assumed to be spherical symmetric. The amount of noncondensable gas in a single bubble is unknown and is strongly dependent on the circumstances. The minimum amount of gas, which can diffuse into a bubble during the growth phase of a bubble, can be estimated to be at least $5 \%$ (volume) for a bubble of radius $0.5 \mathrm{~mm}$. This gives a pressure of $p_{g_{0}}=445 \mathrm{~Pa}$, compared to the vapour pressure of $2000 \mathrm{~Pa}$ at $15^{\circ} \mathrm{C}$ being already a considerable contribution to the total pressure. This contribution becomes more important if the bubble collapses. The velocity of sound in a bubbly mixture, containing mainly vapour bubbles is unknown and will be calculated. Unfortunately, there are almost no data concerning bubble clouds. In the data available the percentages of gas are unknown, yet important according to the present model!

\section{The equations of motion for a cloud of bubbles}

Conservation of mass for a purely vapour filled bubbly cloud gives :

$$
\begin{aligned}
\frac{\partial}{\partial t}\left(\alpha \rho_{v}\right)+\nabla .\left(\alpha \rho_{v} \underline{u}_{v}\right) & =\frac{3 \alpha}{R} \rho_{v}\left(\frac{\partial R}{\partial t}+\underline{u}_{v} \cdot \nabla R\right) \\
\frac{\partial}{\partial t}\left[(1-\alpha) \rho_{l}\right]+\nabla \cdot & {\left[(1-\alpha) \rho_{l} \underline{u}_{l}\right]=} \\
= & -\frac{3 \alpha}{R} \rho_{v}\left(\frac{\partial R}{\partial t}+\underline{u}_{v}, \nabla R\right) .
\end{aligned}
$$

On the right is per unit volume the mass flux due to evaporation and is zero for a purely gas filled cloud. Bubble conservation gives :

$$
\frac{\partial n}{\partial t}+\nabla \cdot\left(n \underline{u}_{,}\right)=0 \quad \text { with } \quad \alpha=n \frac{4}{3} \pi R^{3} .
$$

Conservation of momentum (gas bubbles) BIESHEUvEL et al. [3] gives :

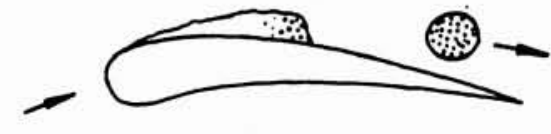

1. The cloud and its surroundings.

$$
\begin{aligned}
& \frac{\partial}{\partial t}\left[(1-\alpha) \underline{u}_{l}\right]+\nabla \cdot\left[(1-\alpha) \underline{u}_{l} \underline{u}_{l}\right]= \\
& \quad-\frac{\nabla}{\rho} \frac{p}{l}-\nabla \cdot\left[\alpha\left\{\left(\frac{\partial R}{\partial t}\right)^{2} \underline{\underline{I}}+\frac{1}{2}\left(\underline{u}_{g}-\underline{u}\right)\left(\underline{u}_{g}-\underline{u}_{)}\right)\right\}\right] .
\end{aligned}
$$

Phase change can be neglected here since the pressure caused by the mass flux through the bubble surface multiplied by the velocity difference is :

$$
p_{\text {mass flux }}=\alpha \frac{\mathrm{d} R}{\mathrm{~d} t} \rho_{v}\left(\underline{u}_{l}-\underline{u}_{v}\right) \leqslant \alpha\left(\frac{\mathrm{d} R}{\mathrm{~d} t}\right)^{2} \rho_{v} \leqslant 10 \mathrm{~Pa}
$$

with

$$
\alpha \leqslant 0.1, \frac{\mathrm{d} R}{\mathrm{~d} t} \leqslant 100 \mathrm{~m} \mathrm{sec}^{-1} \text { and } \rho_{\mathrm{v}} \sim 10^{-2} \mathrm{~kg} \mathrm{~m}^{-3} \text {. }
$$

The equation of motion of the bubbles is the Rayleigh Plesset equation

$$
\begin{gathered}
p_{m}=p+\rho_{l}\left[\frac{3}{2}\left(\frac{\partial R}{\partial t}\right)^{2}+R \frac{\partial^{2} R}{\partial t^{2}}\right] \\
p=(1-\alpha) p_{l}+\alpha p_{m} \text { and } p_{m}=p_{v}+p_{g}
\end{gathered}
$$

with $p$ the averaged pressure of the bubbly mixture. The effect of phase change can be neglected because of the very large density difference between the vapour and the liquid $\left(\rho_{l} \sim 10^{3} \mathrm{~kg} \mathrm{~m}^{-3}\right)$ : the differences between the velocities just outside and just inside the bubble boundary are negligible (PLESSET [4]). The pressure $p_{m}$ consists of a vapour $p_{v}$ and a gas part $p_{g}$ (App. A). The temperature at the bubble boundary is constant (PLESSET [4]), so $p_{v}$ only depends on the velocity of the bubble wall : phase change can't keep up with the rapidly varying bubble radius. Therefore we use the formula used by PLESSET $[4,5]$ based on the mass fluxes going in or out of the bubble boundary :

$$
p_{\mathrm{r}}=p_{r, c_{4}}\left(1+\frac{\partial R}{\partial t} \frac{1}{c^{*}}\right)^{-1}
$$

with

$$
c^{*}=\beta c_{v}(2 \pi \gamma)^{-1 / 2} .
$$

To construct the boundary conditions concerning sound radiation the velocity of sound in a bubbly fluid $\left(c_{0}\right)$ must be known (App. B). In the case of small void fraction, we can simplify our equations considerably by scaling [1]. A typical time scale for our problem is: $t_{0}=A_{0} / c_{0}$. All the velocities are caused by the presence of bubbles, therefore the velocities can all be scaled with 
$\alpha_{0}$ too. The pressures are all scaled with $p_{l 0}\left(=p_{v, \text { eq }}+p_{g 0}\right)$. So :

$$
\begin{array}{rlrlrl}
\underline{u} & =\alpha_{0} c_{0} \underline{\hat{u}} & R=R_{0} \hat{R} & p . . & =p_{l 0} \hat{p} . \\
\alpha=\alpha_{0} \hat{\alpha} & r=A_{0} \hat{r} & \langle T\rangle & =T_{0} \hat{T} \\
\rho_{l}=\rho_{l 0} \hat{\rho}_{l} & \rho_{v}=\rho_{v 0} \hat{\rho}_{v} & t & =t_{0} \hat{t} .
\end{array}
$$

Variables with index 0 are initial values. We can now simplify our equations by retaining only lowest order terms in $\alpha_{0}$.

Bubble conservation :

$$
\hat{n}=1 \Rightarrow \hat{\alpha}=\hat{R}^{3} .
$$

Mass conservation in the liquid for pure ! vapour filled bubbles :

$\frac{\partial \hat{\alpha}}{\partial \hat{t}}=\hat{\nabla} \cdot \hat{\underline{u}}_{l}\left(\right.$ the term with $\rho_{v 0} / \rho_{l 0} \sim 10^{-5}$ is neglected $)$.

For pure! gas filled bubbles we have the same equation [1]! If the bubbles are filled with both gas and vapour, the equation is the same too. The mass equations in the vapour/gas will not be used.

Momentum :

$$
c_{0}^{2} \alpha_{0} \rho_{l 0} p_{l 0}^{-1} \frac{\partial \underline{\hat{\underline{u}}}_{l}}{\partial \hat{t}}=-\hat{\nabla} \hat{p} .
$$

Pressure :

$$
\hat{p}=\hat{p}_{l}
$$

Rayleigh Plesset :

$$
\hat{p}_{m}=\hat{p}_{t}+\kappa\left[\frac{3}{2}\left(\frac{\partial \hat{R}}{\partial \hat{t}}\right)^{2}+\hat{R} \frac{\partial^{2} \hat{R}}{\partial \hat{t}^{2}}\right]
$$

with

$$
\hat{p}_{m}=\hat{p}_{g}+\hat{p}_{v} \quad \text { and } \quad \kappa=R_{0}^{2} \rho_{l} c_{0}^{2} A_{0}^{-2} p_{l 0}^{-1} .
$$

Elimination of $\hat{u}_{l}$ gives us (9), (10), (11):

$$
p_{l 0}\left(c_{0}^{2} \alpha_{0} \rho_{l 0}\right)^{-1} \hat{\nabla}^{2} \hat{p}_{l}+3 \hat{R}^{2} \frac{\partial^{2} \hat{R}}{\partial \hat{t}^{2}}=-6 \hat{R}\left(\frac{\partial \hat{R}}{\partial \hat{t}}\right)^{2} \text {. }
$$

Vapour pressure :

$$
\hat{p}_{v}=\hat{p}_{v, e q}\left(T_{0}\right)\left\{1+\frac{\partial \hat{R}}{\partial \hat{t}} R_{0} c_{0} A_{0}^{-1}\left(c^{*}\right)^{-1}\right\}^{-1} .
$$

Energy (App. A) :

$$
\begin{aligned}
& \tau=9 \hat{\lambda}_{g} \int_{0}^{i} \hat{R} \mathrm{~d} \xi, \quad \hat{\lambda}_{g}=t_{0} \lambda_{g} \rho_{g 0}^{-1} R_{0}^{-2} C_{v g}^{-1} \\
& \hat{p}_{g}=\left(p_{g 0} / p_{l 0}\right) \hat{R}^{-3}\left[1-\frac{8}{\pi^{2}} \sum_{j=0}^{2} \frac{H_{j}(\tau)}{(1+2 j)^{2}}\right]
\end{aligned}
$$

$$
\begin{aligned}
H_{j}(\tau)= & 3 \frac{p_{l 0}}{p_{\theta 0}}(\gamma-1) \exp \left\{-\frac{\pi^{2}}{4}(1+2 j)^{2} \tau\right\} \times \\
& \times \int_{0}^{\tau} \exp \left\{\frac{\pi^{2}}{4}(1+2 j)^{2} x\right\} \hat{p}_{m} \hat{R}^{2} \frac{\partial \hat{R}}{\partial x} \mathrm{~d} x .
\end{aligned}
$$

We will now discuss the boundary and initial conditions. The damping by sound radiation will be represented by the boundary conditions [1]. We can apply a pressure at the boundary of the cloud $\hat{p}_{a}=1+\Delta \hat{p} f(\hat{t})$. Outside the cloud the wave equation is valid, in dimensionless form :

$$
\varepsilon^{2} \frac{\partial^{2} \hat{p}_{l}}{\partial \hat{t}^{2}}-\hat{\nabla}^{2} \hat{p}_{l}=0, \quad \varepsilon=\frac{c_{0}}{c_{l}} .
$$

For spherical symmetric outgoing waves a solution is : $\hat{u}_{l}=\frac{\partial \phi}{\partial \hat{r}}$ with $\phi=F(\hat{t} / \varepsilon-\hat{r}) / \hat{r}$ and $p_{l 0} c_{0}^{-2} \alpha_{0}^{-1} \rho_{l 0}^{-1} \hat{p}_{r a}=$ $-\frac{\partial \phi}{\partial \hat{t}}\left(\hat{p}_{r a}\right.$ is the radiated pressure). Inside the bubble we have $c_{0}^{2} \alpha_{0} \rho_{l 0} p_{l 0}^{-1} \frac{\partial \hat{u}_{l}}{\partial \hat{t}}=-\hat{\nabla} \hat{p}_{l}$. At the cloud boundary $(\hat{r}=1)$ the pressure must be continuous : $\hat{p}_{l}=\hat{p}_{a}+\hat{p}_{r a}$

$\hat{p}_{l}+\frac{\partial \hat{p}_{l}}{\partial \hat{r}}+\varepsilon \frac{\partial \hat{p}_{l}}{\partial \hat{t}}=1+\Delta \hat{p}\left[f(\hat{t})+\varepsilon \frac{\partial f}{\partial \hat{t}}\right], \quad \hat{r}=1$.

Spherical symmetry requires

$$
\frac{\partial \hat{p}_{l}}{\partial \hat{r}}=0 \quad \hat{r}=0
$$

$f(\hat{t})$ is chosen to be a step function at $\hat{t}=0$, the amplitude can be changed with $\Delta \hat{p}$. As initial conditions we have (rest) :

$$
\begin{gathered}
\hat{t}=0: \quad \hat{p}_{l}=\hat{R}=1 \quad \hat{p}_{g}=p_{g 0} / p_{l 0} \quad \hat{p}_{v}=p_{v, e q} / p_{l 0} \\
H_{j}=0 \quad j=1,2,3 \quad \frac{\partial \hat{R}}{\partial \hat{t}}=0 .
\end{gathered}
$$

\section{The numerical method, results and conclusions}

The equations (12)/(17), (19)/(21) are solved numerically. With (12), (13) a matrix equation is set up to solve $\hat{R}$ and $\hat{p}_{l}$ (implicit method); $\hat{p}_{m}$ has to be calculated separately. Integrals are calculated by the trapezoidal rule. The equations determining $\hat{p}_{m}, \hat{R}$ and $\hat{p}_{l}$ are nonlinear, so we have to iterate (fig. 2). The dimensionless cloud radius $\hat{r}$ is subdivided into 100 intervals and one dimensionless time unit into about 1000 steps. The numerical procedure is strongly related to ОMTA [1] with some improvements. The numerical results for a cloud filled with vapour, including some noncondensable gas, are similar to those for a cloud completely filled with gas 


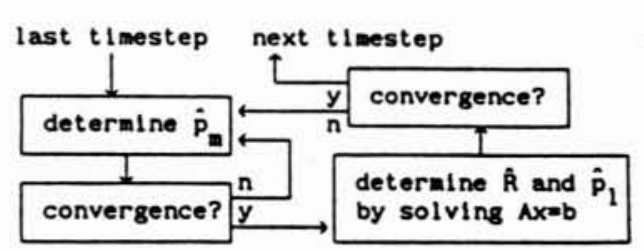

2. The numerical procedure.

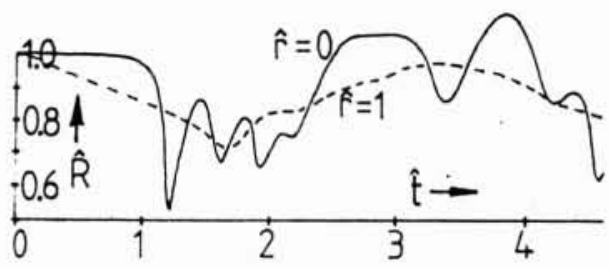

3. $\dot{R}$ vs. $i$ with standard parameters (10\% gas).

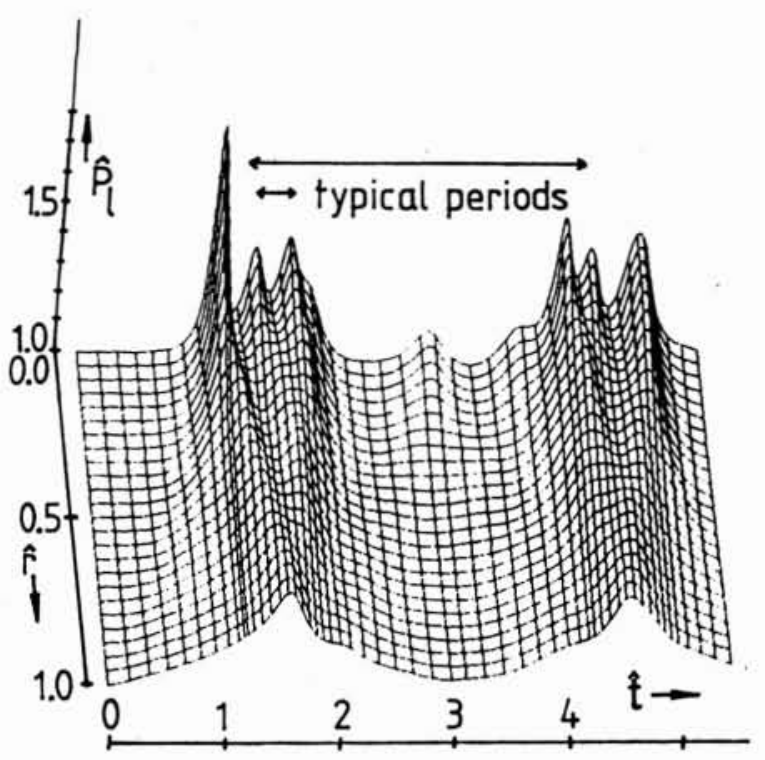

4. $\dot{p}_{l}$ vs. $\dot{r}$ and $\dot{i}$ with standard parameters (10\% gas).

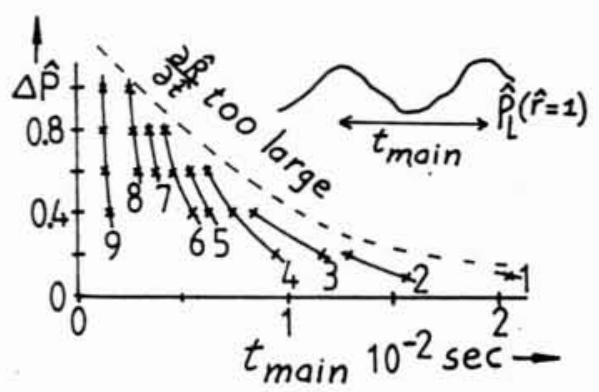

6. Typical(main) period as a function of $p_{g 0}$ and $\left.\Delta \dot{p}: 1\right)$ $p_{g 0}=445 \mathrm{~Pa}(10 \%$ gas $)$; 2) $1094 \mathrm{~Pa}(20 \%)$; 3) $1875 \mathrm{~Pa}$ $(30 \%)$; 4) $2920 \mathrm{~Pa} \quad(40 \%)$; 5) $4375 \mathrm{~Pa} \quad(50 \%)$; 6) $6560 \mathrm{~Pa}(60 \%)$; 7) $10200 \mathrm{~Pa}(70 \%)$; 8) $17500 \mathrm{~Pa}$ $(80 \%)$; 9) $83100 \mathrm{~Pa}(95 \%)$. (the quantitative behaviour however is completely different). Apparently the noncondensable gas becomes important during the final stage of the collapse. In fact, a completely vapour filled bubble shows no rebound at all ! Results will be given for a typical case : $T_{0}=288 \mathrm{~K}$, $\rho_{l}=1000 \mathrm{~kg} \mathrm{~m}^{-3}, \quad A_{0}=0.03 \mathrm{~m}, \quad \alpha_{0}=0.03$, $c_{1}=1465 \mathrm{~m} \mathrm{sec}^{-1}, \quad R_{0}=5.0 \cdot 10^{-4} \mathrm{~m}, \quad \Delta \hat{p}=0.1$, $p_{g 0}=445 \mathrm{~Pa}\left(10 \%\right.$ gas) and $p_{v, e q}=2000 \mathrm{~Pa}$, unless stated otherwise. In figure 4 the disturbance can be seen to move inward. If $\hat{R}$ has a maximum, $\hat{p}_{l}$ has a minimum and vice versa. Two typical periods occur (figs. 3, 4, 5) : one corresponding to the global cloud behaviour (twice the time a signal needs to go from the boundary to the centre of the cloud $\sim 2 t_{0}$; dim. less 2 ) and another to single bubble behaviour (twice the Rayleigh collapse time [7] $\left.t_{R}=\left(\rho_{l} /\left(p_{l 0} \Delta \hat{p}\right)\right)^{1 / 2} 2 R_{0}\right)$. If $\Delta \hat{p}$ becomes too large, $\partial \hat{R} / \partial \hat{t}$ is larger than the velocity of sound in the vapour. Our model is no longer valid in these regions (fig. 6). The low frequency (cloud behaviour) is slightly dependent on $\Delta \hat{p}$, but strongly dependent on $p_{g 0}$ (since

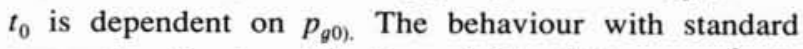
parameters is almost isothermal (fig. 7) because $\hat{\lambda}_{g}$ is proportional to $p_{g 0}^{-3 / 2}\left(t_{0} \sim p_{g 0}^{-1 / 2}\right.$ and $\left.\rho_{g 0} \sim p_{g 0}\right)$. Here $\hat{\lambda}_{g}=1.5 \cdot 10^{2}$. However if $p_{g 0} \sim 1 \mathrm{~atm}$, then $\hat{\lambda}_{g} \sim 10^{-2}$, so the behaviour will be almost adiabatic. In general, if $\hat{\lambda}_{g} \gg 1$ the behaviour is isothermal and if $\hat{\lambda}_{g} \ll 1$, it is adiabatic.

(*) This study is supported by MARIN (Maritime Res. Inst. Netherlands).

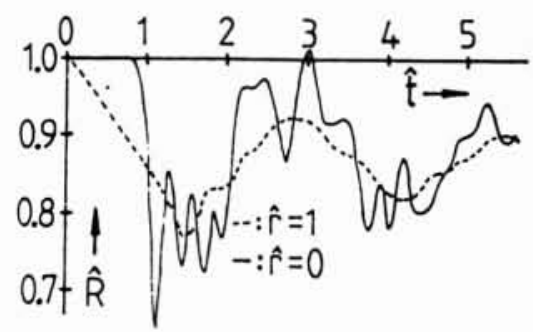

5. $\dot{R}$ vs. $\dot{t}$, standard parameters, but with $p_{g 0}=83100 \mathrm{~Pa}(95 \%$ gas) and $\Delta \hat{p}=0.6$.

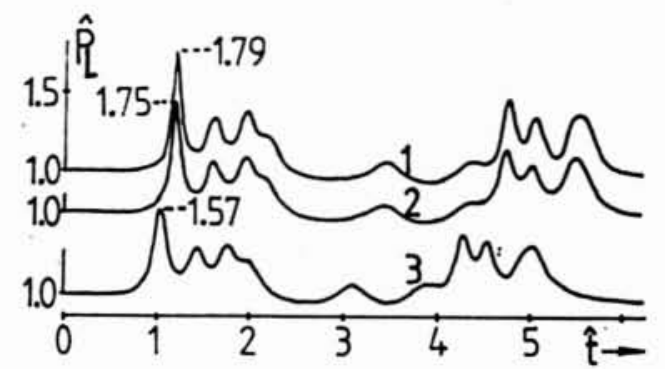

7. Thermal behaviour of the solution with standard parameters : $\dot{p}_{1}$ at $\dot{r}=0$ as a function of $\dot{i}$

1) isothermal ; $\dot{p}_{g}=p_{g} d p_{10} \dot{R}^{-3}$

2) equations $15-17$

3) adiabatic ; $\hat{p}_{g}=p_{g} d p_{l 0} \dot{R}^{-3 \gamma}$. 


\section{Appendix A : calculation of the gas pressure}

For $p_{q}$ we can follow an approach made by FLYNN [6] and OMTA [1] based on the energy equation for the noncondensable gas (thermal damping) :

$$
\rho_{g} C_{v g} \frac{D T}{D t}=-p \nabla \cdot \underline{u}_{g}+\nabla \cdot\left(\lambda_{g} \nabla T\right) .
$$

Time, position and temperature-potential are made dimensionless

$$
y=\rho_{g 0}^{-1} R_{0}^{-3} 3 \int_{0}^{r} \rho_{g} \xi^{2} \mathrm{~d} \xi
$$

$\tau=\rho_{g 0}^{-1} R_{0}^{-3} \lambda_{g} C_{v g}^{-1} 9 \int_{0}^{t} R(\xi) \mathrm{d} \xi \quad C_{v g}, \lambda_{g}$ constant

$\frac{\partial \chi}{\partial y}=-1+T / T_{0}$ with $\chi(y, 0)=0$ and $\chi(0, \tau)=0$.

$$
\begin{array}{rl}
3 \lambda_{g} R T_{0} \frac{\partial \chi}{\partial \tau}=-\int_{0}^{y} & p \frac{\partial}{\partial \xi}\left[u_{g} r^{2}(\xi)\right] \mathrm{d} \xi+ \\
& +3 \lambda_{g} \rho_{g 0}^{-1} R_{0}^{-3} T_{0} r^{4}(y) \rho_{g} \frac{\partial^{2} \chi}{\partial y^{2}} .
\end{array}
$$

We will now make some approximations (OMTA [1]) : the pressure inside the bubble is almost uniform, the density $\rho_{g}$ is almost equal to its averaged value over the bubble $\left(=\rho_{g 0} R_{0}^{3} / R^{3}\right)$. The term $r^{4} / R^{4}$ can be approximated by 1 , since temperature gradients are largest at the bubble boundary. Furthermore, the velocity inside the bubble can be written as : $u_{g} \approx \frac{r}{R} \frac{\mathrm{d} R}{\mathrm{~d} \tau} \frac{\mathrm{d} \tau}{\mathrm{d} t}$. The potential $\chi$ has to satisfy :

$$
\frac{\partial \chi}{\partial \tau}-\frac{\partial^{2} \chi}{\partial y^{2}}=-\left\{3\left(\rho_{g 0} R_{0}^{3} C_{v g} T_{0}\right)^{-1} p R^{2} \frac{\mathrm{d} R}{\mathrm{~d} \tau}\right\} y .
$$

Setting $\quad \chi=W(y, \tau)-\psi(\tau) y \quad$ and noting $\rho_{g 0} T_{0}=$ $p_{g 0}\left(C_{p g}-C_{v g}\right)$ with :

$$
\psi=3(\gamma-1)\left(p_{g 0} R_{0}^{3}\right)^{-1} \int_{0}^{\tau} p(\xi) R^{2}(\xi) \frac{\mathrm{d} R}{\mathrm{~d} \xi}(\xi) \mathrm{d} \xi
$$

results in (since both vapour and gas contribute to $\left.p\left(=p_{v}+p_{g}\right)\right)$ :

$$
\begin{gathered}
\frac{\partial W}{\partial \tau}=\frac{\partial^{2} W}{\partial y^{2}} \quad\left(\frac{\partial W}{\partial y}\right)_{y=1}=\psi(\tau) \\
W(y, 0)=0, \quad W(0, \tau)=0 .
\end{gathered}
$$

The last equation stating the fact that at the bubble boundary the temperature is constant. Solving by Laplace transform results in :

$$
\frac{\partial W}{\partial \tau}=2 \sum_{j=0}^{\infty}(-1)^{j} \sin \{\pi y(1+2 j) / 2\} H_{j}(\tau)
$$

with

$$
H_{j}(\tau)=\mathrm{e}^{-\frac{\pi^{2}}{4}(1+2 j)^{2} \tau} \int_{0}^{\tau} \mathrm{e}^{\frac{\pi^{2}}{4}(1+2 j)^{2} x} \frac{\partial \psi}{\partial x} \mathrm{~d} x
$$

$T / T_{0}$ is related to $W$ by using (A4) and (A8) as : $\frac{\partial T}{\partial y} / T_{0}=\frac{\partial W}{\partial \tau}$.

Now, integration leads to :

$T / T_{0}=1-\frac{4}{\pi} \sum_{j=0}^{\infty}(-1)^{j} \frac{\cos \{(1+2 j) \pi y / 2\}}{(1+2 j)} H_{j}(\tau)$.

Just as in OMTA [1] the mean temperature of the bubble contents is

$$
\begin{aligned}
\langle T\rangle & =3 \rho_{g 0}^{-1} R_{0}^{-3} \int_{0}^{R} T \rho_{g} \xi^{2} \mathrm{~d} \xi= \\
& =\int_{0}^{1} T \mathrm{~d} y=T_{0}\left(1-\frac{8}{\pi^{2}} \sum_{j=0}^{\infty} \frac{H_{j}(\tau)}{(1+2 j)^{2}}\right) .
\end{aligned}
$$

The summation in (A12) can be cut off at $j=2$ because 3 terms are accurate enough for our solution. The gas pressure can finally be written as :

$$
p_{g}=\langle T\rangle / T_{0} p_{g 0}\left(\frac{R_{0}}{R}\right)^{3} .
$$




\section{Appendix B : the velocity of sound in a bubbly mixture}

The density in a bubbly fluid can be written as $\rho=(1-\alpha) \rho_{l}+\alpha \rho_{m}$.

$$
\frac{1}{c_{0}^{2}}=\frac{\mathrm{d} \rho}{\mathrm{d} p}=\frac{\alpha}{c_{m}^{2}}+\frac{(1-\alpha)}{c_{l}^{2}}-\left(\rho_{l}-\rho_{m}\right) \frac{\mathrm{d} \alpha}{\mathrm{d} p} .
$$

With $\alpha=V_{m} / V \quad\left(V_{m}\right.$; the volume of all the bubbles together in $\left.V=V_{l}+V_{m}\right)$ and $1-\alpha=V_{l} / V$; $V_{m}=n V V_{b}\left(V_{b}\right.$ is the volume of a single bubble), $n V$ is the total number of bubbles in $V$ and is assumed to be constant. The total mass of the vapour and the liquid remains $V_{l} \rho_{l}+V_{v} \rho_{v}=$ Const. So with $\rho_{m} \sim 10^{-2} \mathrm{~kg} \mathrm{~m}^{-3}$ and $\rho_{l} \sim 10^{3} \mathrm{~kg} \mathrm{~m}^{-3}$ we get :

$$
\begin{aligned}
\frac{1}{c_{0}^{2}}=\frac{\alpha}{c_{m}^{2}}+ & \frac{(1-\alpha)^{2}}{c_{l}^{2}}-\left(\rho_{l}-\rho_{m}\right)\left\{\frac{(1-\alpha) \alpha}{V_{b}} \frac{\mathrm{d} V_{b}}{\mathrm{~d} p}+\right. \\
& \left.+\frac{\alpha}{V} \frac{\mathrm{d}}{\mathrm{d} p}\left[\frac{V_{v} \rho_{v}}{\rho_{l}}\right]\right\}-\frac{1}{c_{l}^{2}}\left(1-\frac{\rho_{m}}{\rho_{l}}\right) \alpha \frac{V_{v} \rho_{v}}{V \rho_{l}} .
\end{aligned}
$$

Note that: $d\left(\rho_{m} V_{b}\right)=\rho_{m} d V_{b}+V_{b} d \rho_{m} \simeq \rho_{v} d V_{b}$; because the mass of the noncondensable gas remains constant and the vapour pressure is assumed to be almost constant, this leads to :

$$
\begin{aligned}
\frac{1}{c_{0}^{2}}=\frac{\alpha^{2}}{c_{m}^{2}}+\frac{(1-\alpha)^{2}}{c_{l}^{2}}- & \left(\rho_{l}-\rho_{v}\right) \frac{(1-\alpha) \alpha}{V_{b}} \frac{\mathrm{d} V_{b}}{\mathrm{~d} p}- \\
& -\left(1-\frac{\rho_{m}}{\rho_{l}}\right) \frac{\alpha}{V} \frac{\mathrm{d}}{\mathrm{d} p}\left(V_{v} \rho_{v}\right) .
\end{aligned}
$$

The third term can be rewritten using the Rayleigh Plesset equation for small perturbations (YouNG [7]) and $p_{g 0}+p_{v}=p_{t 0}$ (equilibrium):

$$
-\left(\rho_{l}-\rho_{v}\right) \frac{(1-\alpha) \alpha}{V_{b}} \frac{\mathrm{d} V_{b}}{\mathrm{~d} p}=\alpha(1-\alpha) \frac{\rho_{l}}{\gamma p_{g 0}}
$$

The rate of evaporation (4th term) is infinite for an ideal vapour. Multiplying the maximum evaporation per unit time in a single bubble [5] $\beta\left(\frac{\mathcal{R} T}{2 \pi}\right)^{1 / 2} \rho_{v} 4 \pi R^{2}$ by the number of bubbles in $V, \alpha V /\left(\frac{4}{3} \pi R^{3}\right)$, gives us :

$$
\begin{aligned}
\frac{\alpha}{V} \frac{\mathrm{d}}{\mathrm{d} p}\left(V_{v} \rho_{v}\right)=\frac{\alpha}{V} & \frac{\mathrm{d}}{\mathrm{d} t}\left(V_{v} \rho_{v}\right) / \frac{\mathrm{d} p}{\mathrm{~d} t} \leqslant \\
& \leqslant \beta(\gamma 2 \pi)^{-1 / 2} c_{v} \rho_{v} \frac{3}{R} \alpha^{2} / \frac{\mathrm{d} p}{\mathrm{~d} t} .
\end{aligned}
$$

With $\quad \rho_{m} / \rho_{l} \sim 10^{-5}, \quad \alpha \sim 10^{-2}, \quad \beta(\gamma 2 \pi)^{-1 / 2} \sim 1$, $c_{v} \sim 300 \mathrm{~m} \mathrm{sec}^{-1}, R \sim 10^{-3} \mathrm{~m}, \rho_{v} \sim 10^{-2} \mathrm{~kg} \mathrm{~m}^{-3}$ and the pressure change at least 1 pascal within a typical bubble collapse time $\left(10^{-3} \mathrm{sec}\right)$, the last term in (B2) can be neglected. So only the third term in (B2) remains, leading to a sound velocity in a mixture dominated by the presence of noncondensable gas

$$
c_{0}^{2} \approx \frac{p_{g 0} \gamma}{\rho_{l} \alpha_{0}} .
$$

\section{References}

[1] Омта R. - Oscillations of a Cloud of Bubbles of Small and not so Small Amplitude, J. Acoust. Soc. Am. 82, 1018-1033 (1987).

[2] Prosperetti A., Crum L.A., Commander K.W. - Nonlinear Bubble Dynamics, J. Acoust. Soc. Am. 83(2), 502-514 (1988).

[3] Biesheuvel A. and van Wungaarden L. - Two-phase Flow Equations for a Dilute Dispersion of Gas Bubbles in Liquid, J. Fluid Mech. 148, 301-318 (1984).

[4] Plesset M.S. - Bubble Dynamics (in Cavitation in Real Liquids, edited by R. Davies) 9-18 (1964).

[5] Plesset M.S., Prosperetti A. - Flow of Vapour in a Liquid Enclosure, J. Fluid Mech. 78, 433-444 (1976).

[6] FlynN H.G. - Cavitation Dynamics, 1. A Mathematical Formulation, J. Acoust. Soc. Am. 57, 1379-1396 (1975).

[7] Young F.R. - Cavitation, McGraw-Hill, London, 9-37, 164-168 (1989). 